Национальный.медицинский исследовательский центр онкологии

им. Н.Н. Блохина Минздрава РФ (Москва, Россия)

Российский университет дружбы народов (Москва, Россия)

\section{ЧТО ИЗМЕНИЛОСЬ}

\section{В КЛИНИЧЕСКОЙ ПРАКТИКЕ В ОНКОЛОГИИ В 2019 ГОДУ?}

М.Ю. Федянин

WHAT HAS CHANGED IN CLINICAL ONCOLOGY PRACTICE IN 2019?

В статье на примере злокачественных опухолей желудочно-кишечного тракта рассматриваются изменения лечебных подходов в 2019-2020 годах. Как вести пациента с операбельным раком желудка, что эффективнее - периоперационная или послеоперационная химиотерапия? Какова истинная эффективность иммунотерапии и возможно ли снижать интенсивность лечения больных с диссеминированными опухолями желудка. Произошла ли персонификация лечения при раке поджелудочной железы? Какой новый стандарт первой линии распространенного гепатоцеллюлярного рака? Критически рассмотрим исследования при операбельном и распространенном раке толстой кишки.

Ключевые слова: рак, химиотерапия, иммунотерапия, таргетная терапия.

The article focused on the changes in the treatment approaches in patients with the gastrointestinal malignances in 2019-2020. How to treat a patient with operable gastric cancer, what is more effective - perioperative or postoperative chemotherapy? What is the true effectiveness of immunotherapy and is it possible to reduce the intensity of treatment for patients with advanced gastric cancer. Did we achieve a personification of treatment for pancreatic cancer? What is the new standard of the first-line for advanced hepatocellular cancer? We critically examine studies in early and advanced colon cancer.

Keywords: cancer, chemotherapy, immunotherapy, targeted therapy. 
$\mathrm{K}$ ждый год сообщаются результаты сотен рандомизированных исследований о злокачественных опухолях. Только часть из них действительно меняют клиническую практику. Учитывая объем информации, ежегодно можно выпускать отдельную монографию о событиях, которые изменили лечебные подходы в онкологии. И действительно, если посмотреть на динамику страниц в рекомендациях по лечению солидных опухолей общества RUSSCO, то данное руководство увеличилось со 192 страниц в 2012 году до 776 в 2019 году. Тем самым, учитывая ограничение статьи по объему, в данном обзоре критически рассмотрим, что значимо изменилось в клинической практике в 2019 году на примере опухолей желудочно-кишечного тракта.

\section{Рак желудка}

К 2019 году устоявшимися стандартами ведения больных операбельным раком желудка явилось проведение периоперационной химиотерапии режимом FLOT, начиная со стадии T2N0M0 [1], или проведение адъювантной химиотерапии по фторпиримидинами с или без оксалиплатина [2-5]. Но сохранялись вопросы: нужен или нет оксалиплатин в адъювантном лечении и что эффективнее - периоперационная или послеоперационная химиотерапия.

На первый вопрос ответило исследование ARTIST2. B ней, кроме того, что был забит очередной гвоздь в проведение химиолучевой терапии при резектабельном раке желудка, авторы показали значимое улучшение показателей выживаемости без признаков болезни при применении адъювантно режима SOX (S1 и оксалиплатин) в течение 6 месяцев, в сравнении с монотерапией S1 в течение 1 года (OP 0,648, 95\% ДИ 0,467-0,898, p=0,009) [6].

На второй вопрос в 2019-2020 годах отвечало уже несколько работ. Kang с соавторами изучили эффективность периоперационного подхода, когда до операции назначался режим - доцетаксел, оксалиплатин и S1 (3 курса), а после операции пациенты переводились на монотерапию препаратом S1 (8 курсов). В качестве контрольной группы выступали пациенты, которым после хирургического лечения на первом этапе адъювантно назначался S1 (8 курсов). В исследование включено 530 больных раком желудка стадиями Т2-3N+M0 и T4N любое M0. В качестве основного критерия әффективности выступал показатель 3-летней выживаемости без признаков болезни. В группе послеоперационной химиотерапии была выше частота R0 резекций $(96,4 \%$ против $85,8 \%$, $\mathrm{p}<0,0001)$, не отмечено влияния предоперационного этапа ни на частоту послеоперационных осложнений, ни на процент больных, которые закончили 8 курсов адъювантной химиотерапии (83,3\% против $84 \%$, соответственно). У 10,4\% пациентов достигнут полный патоморфологический эффект, значимо уменьшилась патоморфологическая стадия болезни, что привело к увеличению 3-летней выживаемости без признаков болезни на 6,1\% (ОР 0,7, 95\% ДИ 0,52-0,95, p=0,023). Показатели общей выживаемости не различались, так как зарегистрировано пока только 29,3\% и 27,3\% событий в сравниваемых группах [7]. Аналогичные данные по частоте снижения стадии, частоте R0 резекций и частоте полных патоморфологических эффектов достигнуты и в другом рандомизированном проспективном исследовании III фазы, но уже из Китая, в котором исследователи сравнили назначение 2-4 курсов режима SOX до и после операции и адъювантного назначения данного режима у пациентов II-III стадии [8].

Ji с соавторами сравнил 3 лечебных подхода: 1 группа - операция и адъювантное назначение режима XELOX (8 курсов), 2 группа - адъювантное назначение режима SOX (8 курсов) и 3 группа - периоперационное назначение режима SOX (3 курса до операции, 5 курсов после и дополнительно еще 3 курса монотерапии S1). Исследователи рандомизировали 1094 пациента со стадией T4aN+M0 или T4bN любое М0. Основным критерием әффективности явилась 3-летняя выживаемость без признаков болезни. Авторы доказали не меньшую эффективность адъювантного назначения режима SOX режиму XELOX по показателю 3-летней выживаемости без признаков болезни (54,8\% против 60,3\%, соответственно; ОР 0,85, 95\% Ди 0,67-1.07, p=0,162). Периоперационное назначение режима SOX не увеличило частоту выполнения операций в сравнении с адъювантным назначением режимов SOX и XELOX - 85,5\%, 92,7\% и 90,4\%, соответственно. Несмотря на это, показатели 3-летней выживаемости без признаков болезни составили: 62,02\%, 60,29\%, 54,78\%, соответственно (ОР 0,79, 95\% Ди 0,62-0,99, p=0,045 при сравнении периоперационного режима SOX и адъювантной схемы XELOX) [9].

Таким образом, если нет возможности назначить периоперационное лечение при относительно местно-распространенных резектабельных опухолях желудка, то в послеоперационном периоде предпочтение лучше отдавать режиму с включением фторпиримидинов и оксалиплатина. Тем не менее, несмотря на азиатскую популяцию, возможно, при данных за поражение регионарных лимфоузлов при сТ2-Т4 или T4N0, в обязательном порядке рассматривать назначение периоперационного лечения.

При распространенном раке желудка наиболее значимым, с точки зрения практической онкологии, явились результаты исследований не по применению иммунотерапии до, после или вместо химиотерапии, а исследование по оптимальному дозовому режиму схемы XELOX у ослабленных и пожилых пациентов. Авторы работы рандомизировали 514 больных раком желудка, способных перенести режим XELOX, на 3 группы: в 1 группе - доза оксалиплатина составила 130 мг/м² в 1 день, доза капецитабина - 1250 мг/м² в сутки 1-21 дни, во 2 группе - редукция доз составила 20\%, 
в 3 группе 40\% от доз 1 группы. Авторы доказали не меньшую эффективность по показателю 1-годичной выживаемости без прогрессирования как 2 группы (20\% редукции), так и 3 группы (40\% редукции) 1 группе пациентов (100\% дозы): ОР 1,09, 95\% ДИ 0,89-1,32 и OP $1,1,95 \%$ ДИ 0,9-1,33, соответственно. Аналогично и не различались медианы продолжительности жизни: 6,$7 ; 7,6$ и 7,5 месяцев, соответственно. При этом в 3 группе (40\% редукции доз) у клинически значимо большего процента пациентов сохранялось хорошее самочувствие на 9 неделе лечения в сравнении с группой $1-43 \%$ и 35\%, соответственно. Эти позитивные находки относятся и к качеству жизни, и токсичности и длительности терапии при применении сниженных доз [10]. Таким образом, ослабленным и пожилым пациентам (старше 70-75 лет) можно рассматривать назначение редуцированных двойных комбинаций. Почему же не монотерапии капецитабином? Потому, что в ранее опубликованном небольшом рандомизированном исследовании Hall с соавторами показал улучшение выживаемости при применении двойных комбинаций в сравнении с монотерапией у пожилых и ослабленных пациентов с распространенным раком желудка в первой линии. Медианы продолжительности жизни составили 9,5 против 3,6 месяцев, соответственно [11].

В контексте возможности снижения токсичности лечения при метастатическом раке желудка интересны результаты исследования SOX-GC, в котором авторы доказали, что применение режима комбинации S1 и оксалиплатина в первой линии диффузного или смешанного типа не только менее токсично в сравнении с режимом S1 с цисплатином, но и более әффективно, как в отношении медианы выживаемости без признаков болезни (5,7 против 4,9 месяцев, ОР $0,75,95 \%$ ДИ $0,63-0,89, \mathrm{p}<0,01)$, так и продолжительности жизни (13 против 11,8 месяцев, ОР 0,76, 95\% ДИ 0,63-0,91, p<0,001) [12]. А если вспомнить преимущество режима ЕОХ над режимами EOF/ECX/ECF еще в исследовании Cunningham с соавторами в 2008 году [13], то о применении цисплатина в лечении диссеминированного рака желудка можно смело забыть.

Кроме этого, попытки интенсификации первой линии терапии при распространенном раке желудка пока терпят неудачу. Ранее в 2018 года были представлены негативные результаты исследования III фазы по добавлению доцетаксела к режиму CS (цисплатин c S1) в первой линии терапии распространенного рака желудка. Одногодичная общая выживаемость в группе с доцетакселом составила 59,7\%, а без него $61,5 \%$ (ОР 0,99, 95\% ДИ 0,85-1,16, p=0,47) [14]. А в 2013 году Al-Batran с соавторами доказал неэффективность применения режима FLOT у пациентов старше 65 лет в сравнении с режимом FLO - медиана продолжительности жизни составила 17,3 месяца против 14,5 месяцев $(\mathrm{p}=0,39)$, при значимо большей частоте осложнений 3-4 степени (81,9\% против 38,6\%, соот- ветственно) [15]. Также можно вспомнить и результаты рандомизированного исследования II фазы 2019 года, в котором добавление паклитаксела к режиму FOLFOX увеличило медиану выживаемости без прогрессирования (6,444 против 4,077 месяцев, ОР 0,569, 95\% ДИ $0,326-0,991, \mathrm{p}=0,043)$, но значимо не влияло на продолжительность жизни (9,534 против 6,641 месяцев, ОР 0,729, 95\% ДИ 0,4-1,328, p=0,3) [16]. Таким образом, перед внедрением в повседневную практику в первую линию лечения больных метастатическим раком желудка интенсифицированных режимов - FLOT, FOLFIRINOX и т. п. - необходимо дождаться результатов рандомизированных исследований III фазы.

Исследование ANGEL подтвердило необходимость дожидаться результатов проверочных исследований, если первая работа выполнена в Китае или азиатских стран и касается метастатического рака желудка. Так ранее были доложены результаты исследования на китайской популяции химиорефрактерных пациентов тирозинкиназного ингибитора с высокой аффинностью к VEGFR2 апатиниба. Исследователи показали значимое увеличение медианы продолжительности жизни в сравнении с плацебо: 6,5 месяцев против 4,7 месяцев (ОР 0,709, 95\% ДИ 0,537-0,937, $\mathrm{p}=0,0156$ ) [17]. В исследовании же ANGEL на аналогичной популяции, но с включением не только стран Азии, но и США и Европы, удалось увеличить лишь выживаемость без признаков болезни (2,8 месяцев против 1,8 месяцев, ОР $0,57,95 \%$ ДИ 0,46-0,79, p<0,0001), но не продолжительность жизни (5,8 против 5,1, ОР 0,93, 95\% ДИ $0,74-1,15, \mathrm{p}=0,485)[18]$.

Аналогично, с осторожностью, следует относиться и к результатам исследований по применению иммунотерапии при распространенном раке желудка. Так, если монотерапия ниволумабом в $>2$ линии терапии распространенного рака желудка в азиатской популяции пациентов скромно, но статистически значимо увеличило медиану продолжительности жизни больных в сравнении с плацебо (5,26 против 4,14 месяцев, ОР 0,63, 95\% ДИ 0,41-0,78, p<0,0001) [19], то применение в аналогичных условиях антиPD-L1 антитела (авелумаба) в сравнении с терапией на выбор исследователя или анти-PD-1 антитела пембролизумаба в сравнении с паклитакселом, было не эффективным (ОР 1,1, 95\% ДИ 0,9-1,4, p=0,81 и ОР $0,82,95 \%$ ди 0,66-1,03, p=0,0421 (необходимо было $0,0135)$, соответственно) [20,21]. Негативно на выживаемость без прогрессирования влияло и назначение монотерапии анти-CTLA-4 антитела ипилимумумаба в сравнении с наилучшей симптоматической терапией (2,92 против 4,9 месяцев, соответственно, ОР $1,44,80 \%$ ди 1,09-1,91, p=0,097) [22]. Нужно отдать должное, что в исследовании пембролизумабом в группе с выраженной экспрессией PD-L1 (CPS $\geq 10)$, отмечался выигрыш в выживаемости (ОР 0,64, 95\% Ди 0,41-1,02). Несмотря, что это был поданализ исследования, аналогичные данные получены и в 
работе по применению пембролизумаба во 2 линии распространенного рака пищевода (ОР 0,69, 95\% Ди 0,52-0,92, p=0,0074) [23]. Поэтому, если вы и рассматриваете назначение пембролизумаба при раке пищевода или раке желудка у химиорефрактерных пациентов, то желательно ограничиться популяцией с CPS $\geq 10$. По крайней мере, именно в этой группе нет пересечения графиков выживаемости на раннем этапе, и вы не навредите пациентам.

Попытки перевода иммунотерапии на поддерживающий этап после достижения контроля болезни после 12 недель первой линии лечения с включением оксалиплатина и фторпиримидинов не привело к увеличению продолжительности жизни. Примером могут служить результаты исследования JAVELIN Gastric 100: в группе с поддерживающей терапией авелумабом медиана продолжительности жизни составила 10,4 месяцев против 10,9 месяцев в группе продолжения химиотерапии или наблюдения (OP 0,91, 95\% ДИ 0,74-1,11, p=0,1779) [24]. В исследовании PLATFORM предварительный анализ также показал преимущество поддерживающей терапии капецитабином в сравнении с наблюдением или анти-PD-L1 антителом дурвалумабом - 12-недельный показатель выживаемости без прогрессирования составил 56\%, 49\% и 48\%, соответственно [25].

В исследовании KEYNOTE-062 добавление пембролизумаба к химиотерапии первой линии также не увенчалось успехом или, говоря словами статистики, первичная конечная точка (улучшение общей выживаемости) не была достигнута: ни при CPS $\geq 1$ (ОР 0,85, 95\% ДИ 0,7-1,03, p=0,0046), ни при CРS $\geq 10$ (OP $0,85,95 \%$ ДИ 0,62-1,17, p=0,158). Была надежда, что монотерапия пембролизумабом будет не менее эффективной опцией химиотерапии. Однако полученные результаты трактуются неоднозначно. Авторы исследования говорят, что неменышая эффективность в отношении общей выживаемости доказана, как при значениях СРS $\geq 1$ (ОР 0,91, 95\% ДИ 0,69-1,18, граница неменьшей эффективности 1,2), таки и при CPS $\geq 10$ (ОР 0,69, 95\% ДИ 0,49-0,97). Но, если посмотреть на результаты выживаемости без прогрессирования, то при значениях CPS $\geq 1$ увидим значимое ухудшение данного показателя в группе монотерапии пембролизумабом (ОР 1,66, 95\% ДИ 1,37-2,01). При CPS $\geq 10$ - данное различие не столь удручающе (ОР 1,1, 95\% ДИ 0,79-1,51) [26]. Полученные данные, а также фармакоэкономические показатели не позволяют рекомендовать данную опцию в рутинной клинической практике всем пациентам.

Необходим поиск биомаркеров для терапии ингибиторами иммунных контрольных точек при раке желудка. Так поданализ исследования KEYNOTE-062 в зависимости от наличия микросателлитной нестабильности высокого уровня, четко показывает, что уже в первой линии монотерапия пембролизумабом значимо улучшает показатели выживаемости в срав- нении со стандартной химиотерапией при MSI-H (одногодичная общая выживаемость при CPS $\geq 1$ составила 79\% против 47\%, соответственно, ОР 0,29, 95\% Ди 0,11-0,81) [26]. Аналогичные результаты прослеживаются и в других работах $[27,28]$.

\section{Гепатоцеллюлярный рак}

Продолжая тему иммунотерапии, нельзя не коснуться и лечения распространенного гепатоцеллюлярного рака. Ранее и пембролизумаб и ниволумаб были зарегистрированы во 2 и последующих линиях терапии независимо от экспрессии PD-L1 на основании результатов нерандомизированных исследований I/II фаз при данной патологии. Оба антитела продемонстрировали схожую эффективность - частота объективных эффектов составила $17 \%$ и $15 \%$, соответственно [29, 30]. Тем не менее, все ожидали результатов рандомизированных исследований. Первыми представляли результаты сравнения пембролизумаба и плацебо у пациентов, которым ранее проводилась терапия сорафенибом. В исследование было включено 413 больных. Основной критерий эффективности - общая выживаемость. Частота объективных эффектов в группе с пемролизумабом составила 18,3\%. Отмечено улучшение медианы и выживаемости без прогрессирования и продолжительности жизни в группе анти-PD-1 антитела: 3 и 13,9 месяцев против 2,8 и 10,6 месяцев, соответственно (ОР $0,718,95 \%$ ДИ $0,57-0,904, \mathrm{p}=0,0022$ и ОР $0,781,95 \%$ ди $0,611-0,0238, \mathrm{p}=0,0238)$. Однако с точки зрения статистики исследование считается не достигшим ожидаемого первичного критерия эффективности, так как вероятность ошибки I рода при данной выборке пациентов должна была составлять 0,0174 . Тем не менее, данную работу можно считать негативной, но с позитивными выводами, так как все же эффективность пембролизумаба была подтверждена.

Во второй работе, уже с ниволумабом, исследователи сразу решили пойти в первую линию терапию и улучшить результаты лечения в сравнении с сорафенибом при распространенном гепатоцеллюлярном раке. Было рандомизировано 743 пациента с ECOG 0-1 и классом А по Чайлд-Пью. Основным критерием эффективности явилась общая выживаемость. В группе ниволумаба увеличилась частота объективных әффектов до 15\% против 7\% в группе сорафниба. Однако значимо улучшить медиану продолжительности жизни не удалось: 16,4 против 14,7 месяцев, соответственно (ОР 0,85, 95\% ДИ 0,72-1,02, p=0,0752) [31]. Таким образом, тирозинкиназные ингибиторы (сорфаниб и ленватиниб) оставались до ноября 2019 года стандартом первой линии терапии распространенного гепатоцеллюлярного рака. Но уже на конгрессе ESMO-ASIA 2019 были представлены результаты исследования по сравнению эффективности комбинации атезолизумаба (анти-PD-L1 антитела) и бевацизумаба против сорафениба в первой линии 
терапии больных распространенным гепатоцеллюлярным раком с ECOG 0-1 и классом А по Чайлд-Пью. Был рандомизирован 501 пациент. Основных критерия эффективности было два - выживаемость без прогрессирования и общая выживаемость. Авторам исследования удалось доказать улучшение медианы и выживаемости без прогрессирования и продолжительности жизни в пользу комбинации: 6,8 месяцев и не достигнута против 4,3 и 13,2 месяцев, соответственно (ОР $0,59,95 \%$ ДИ $0,47-0,76, \mathrm{p}<0,0001$ и ОР $0,58,95 \%$ ди $0,42-0,79, \mathrm{p}=0,0006)$. Также увеличилась и частота объективных эффектов с 12\% до 27\%. При этом комбинация показывала меньшую частоту осложнений 3-4 степени, связанных с лечением: 36\% против 46\% [32] и улучшала качество жизни в сравнении с сорафенибом [33]. Таким образом, сформировался новый подход в первой линии терапии распространенного гепатоцеллюлярного рака. Однако для пациентов РФ данный стандарт не успел войти в рекомендации Минздрава.

\section{Рак поджелудочной железы}

В химиотерапии рака поджелудочной железы хоть и произошли изменения за последние 5 лет, но к 2019 году были определены стандарты как адъювантного лечения, так и лечебной химиотерапии. Так в послеоперационном лечении при сохранном функциональном статусе, уровне СА-19-9 менее 180 Ед/мл и R0-R1 резекции, наилучшие результаты по показателям общей выживаемости были достигнуты при применении 12 курсов терапии по схеме mFOLFIRINOX (медиана продолжительности жизни 54,4 месяца) [34], на втором месте идет комбинация капецитабина и гемцитабина (эффективная только при R0 резекции - медиана продолжительности жизни - 39,5 месяцев) [35], при невозможности назначения первых двух режимов - монотерапия гемцитабином или фторпиримидинами в течение 6 месяцев (медиана продолжительности жизни - 22,1 и 17,9 месяцев, соответственно) [36-38].

В 2019 году представлены результаты исследования АРАСТ по сравнению комбинации наб-паклитаксела и гемцитабина и монотерапии гемцитабина в адъювантном назначении при раке поджелудочной железы. В исследование было включено 866 пациентов после R0-R1 резекции в хорошем соматическом статусе при уровне Са-19-9 <100 Ед/мл. Исследователям не удалось улучшить основной критерий эффективности - выживаемость без признаков болезни, оцененная независимым рентгенологом: 19,4 месяцев в группе комбинации против 18,8 месяцев в группе монотерапии гемцитабином (ОР $0,88,95 \%$ ДИ $0,729-$ $1,063, \mathrm{p}=0,1824)$. При этом по результатам предварительного анализа исследователям удалось увеличить медиану продолжительности жизни при добавлении набпаклитаксела с 36,2 до 40,5 месяцев (ОР 0,82, 95\% Ди 0,68-0,996, p=0,045) [39]. Тем не менее, учитывая схожесть критериев включения с исследованием по адъювантному назначению mFOLFIRINOX, принимая во внимание стоимость режима с наб-паклитакселом, отсутствие увеличения выживаемости без признаков болезни в исследовании АРАСТ, возможном влиянии на показатели общей выживаемости различных прогностических факторов и лечения после прогрессирования, в настоящее время не следует рекомендовать комбинацию наб-паклитаксела и гемцитабина в адъювантной терапии больных раком поджелудочной железы.

Рассматривая терапию метастатического или местно-распространенного рака поджелудочной железы, то по-прежнему основные терапевтические опции первой линии ограничены назначением режимов FOLFOXIRI [40], комбинации наб-паклитаксела и гемцитабина и монотерапии гемцитабином, в зависимости от функционального статуса пациента [41]. Учитывая токсичность режима FOLFIRINOX, несмотря на необходимость вести пациента до прогрессирования, в классическом исследовании имеется фраза, что при достижении объективного эффекта рекомендуется проведение 6 месяцев терапии (12 курсов). При этом среднее число курсов составило 10. В 2018 году представили результаты исследования PRODIGE 35-PANOPTIMOX, в котором сравнили продолжение режима FOLFOXIRI до 6 месяцев, с 4 месяцами FOLFOXIRI с последующим переходом на поддерживающую терапию инфузиями лейковорина и фторурацила или с чередованием режимов гемцитабина и FOLFIRI3 каждые 2 месяца. В исследование было рандомизировано 273 пациента. Частота объективных эффектов составила: $35 \%, 41 \%$ и $17 \%$, соответственно. При этом медианы выживаемости без прогрессирования и продолжительности жизни составили: 6,3 и 10,1, 5,7 и 11,2, 4,5 и 7,3 месяцев, соответственно. Авторы работы пришли к выводу, что можно после 8 курсов FOLFIRINOX переходить на поддерживающую терапию, а не оставлять больных без лечения [42].

В этой связи неоднозначно трактуются результаты другого исследования по поддерживающей терапии олапарибом после 6 месяцев терапии первой линии с включением препаратов платины при распространенном раке поджелудочной железы, ассоциированным с мутациями в генах BRCA1 и 2. Одним из основных критериев включения в исследование явилось положение об отсутствии прогрессирования после 16 недель терапии первой линии. При этом в контрольной группе эффективное лечение прекращали и переводили пациентов на плацебо, тогда как в исследуемой группе - на PARP ингибитор. В качестве критерия эффективности приняли не общую выживаемость, а выживаемость без прогрессирования на поддерживающем лечении, увеличив данный показатель в исследовании с 3,8 до 7,4 месяцев (ОР 0,53, 95\% ДИ $0,35-0,82, \mathrm{p}=0,004)$. При этом медианы продолжительности жизни между группами не различались - 18,1 против 18,9 месяцев, соответственно (ОР 0,91, 95\% ДИ 
0,56-1,46, p=0,68) [43]. Таким образом, в отсутствии адекватной группы контроля и отсутствии улучшения общей выживаемости, переходить на поддерживающее лечение олапарибом в данной клинической ситуации рано.

В продолжение данной темы интересны и результаты исследования O'Reilly с соавторами, представленного на конференции ASCO GI 2020. Исследователи рандомизировали 50 больных с распространенным раком поджелудочной железы с мутациями в генах BRCA1, BRCA2 и PALB2 на терапию по схеме цисплатин с гемцитабином с или без PARP ингибитора велипариба. В качестве основного критерия эффективности выбрана частота объективных эффектов. Авторы работы сообщили об отсутствии значимых различий в частоте объективных эффектов между группой с велипарибом и без него - 74,1\% против $65,2 \%$, соответственно. Также не различались и медианы выживаемости без прогрессирования и продолжительности жизни - 10,1 и 15,5 месяцев и 9,7 и 16,4 месяцев, соответственно [44]. Выводами данных работ является необходимость изучения у пациентов перед началом лечения мутационного статуса генов гомологичной рекомбинации, в частности BRCA1 и 2 и PALB2 (секвенировав все 3 гена), и, в случае выявления мутации, назначения препаратов платины. Tерапия же PARP ингибиторами может проводиться в химиорефрактрных состояниях и в рамках клинических исследований.

\section{Рак толстой кишки}

\section{Химиолучевая терапия}

Работа, представленная исследователями из Франции, была посвящена сравнению сроков реализации әффекта химиолучевой терапии (ХЛТ) при местнораспространенном раке прямой кишки. Напомним, что классическим вариантом ведения пациентов с местно-распространенным раком прямой кишки является проведение предоперационной ХЛТ с последующим, через 6-8 недель с момента окончания ХЛТ, хирургическим лечением. Данная рекомендация по срокам ожидания реализации эффекта ХЛТ основана на результатах исследования Lyon R90-01, в котором авторы выявили значимое уменьшение опухоли в размерах при длительном интервале (6-8 недель) в сравнении с группой с коротким интервалом времени до операции (2 недели): $71,7 \%$ против $53,1 \%, \mathrm{p}=0,007$ [45]. Более того, по результатам более современного исследования Probst с соавторами, хотя и ретроспективного, на выборке в 17255 больных, частота полных патоморфологических эффектов была значимо выше при интервале между двумя методами лечения более 8 недель, в сравнении с интервалом в 6 недель - 13,2\% против 8,7\%, p<0,001 [46]. Аналогичные находки описаны и в отношении короткого курса предоперационной лучевой терапии (5х5 Гр) [47]. Выявленные закономерности особенно актуальны в контексте возможности наблюдения за пациентами, у которых достигнут полный клинический эффект [48]. В связи, с чем, в надежде достижения полного клинического эффекта, во многих клиниках стали ожидать реализации эффекта ХЛТ и 3 и 4 месяца, заполнять этот интервал несколькими курсами химиотерапии, а иногда и всеми 8 курсами режима XELOX. Однако Probst c соавторами уже было показано, что, после 11 недель ожидания частота достижения полного патоморфологического эффекта выходит на плато приблизительно в $11 \%$. Более того, увеличение интервала более 7 недель не приводит к значимому увеличению частоты органо-сохранных операций (92,4\% против $89,9 \%, \mathrm{p}=0,078)$, это также не влияло на безрецидивную и общую выживаемость [49].

Все перечисленное делало актуальным проведение проспективной рандомизированной оценки необходимости удлинения интервала с 7 до 11 недель. Критериями включения в исследование, озвученное на ASCOGI 2019, явились больные с местно-распространенным раком прямой кишки (Т3/T4 или $\mathrm{TxN}+\mathrm{M} 0)$ с нижне- и среднеампулярной локализацией. Пациентам проводилась ЛТ (СОД 50 Гр) на фоне внутривенного введения 5-фторурацила. В качестве основного критерия эффективности авторы работы выбрали показатель частоты полных патоморфологических регрессий. В исследование было рандомизированно 267 пациентов. Из них 253 выполнено хирургическое лечение в объеме ТМЕ. Частота полных патоморфологических эффектов для всей популяции рандомизированных больных, в группе 7 недель составила 15\%, а в группе 11 недель - 17,4\%, p=0,5983; в популяции больных, которым выполнено хирургическое лечение - $17,2 \%$ и 15,7\%, соответственно, $p=0,78$. Также длительность интервала не влияла ни на безрецидивную, ни на общую выживаемость пациентов. Более того не оправдались надежды по увеличению частоты полных эффектов отдельно в группе с нарастающим клиническим ответом на ХЛТ при увеличении интервала наблюдения. Авторы исследования пришли к выводу, что удлинение интервала с 7 до 11 недель между окончанием ХЛТ и хирургическим этапом: не приводит к увеличению частоты достижения урT0N0M0; не влияет на БРB и OB; не влияет на частоту рецидивов и метастазирование; при хорошем ответе на ХЛТ удлинение интервала не дает никаких преимуществ; в отсутствии стратегии по отказу от хирургии, операция можно выполнять в сроки 7-8 недель с момент завершения ХЛТ [50].

\section{Адбювантная терапия}

В 2017 году исследователи программы IDEA доказали возможность уменьшения числа курсов адъювантной химиотерапии при III стадии рака ободочной кишки в группе благоприятного прогноза (T1-3N1M0) до 4 курсов XELOX, без компрометации показателей 3-летней выживаемости без признаков болезни. Од- 
нако можно было или нет, полученные результаты транслировать на пациентов со II стадией болезни с факторами риска было неизвестно [51]. Тем более что при III стадии болезни T4N1M0 уменьшение числа курсов комбинации оксалиплатина и фторпиримидинов было ассоциировано с меньшими показателями выживаемости. Тем актуальнее стали результаты, опубликованного в 2019 году совокупного анализа исследований по сравнению длительности адъювантной химиотерапии в течение 3 и 6 месяцев комбинацией оксалиплатина и фторпиримидинов при осложненной II стадии рака толстой кишки программы IDEA. Принимая во внимание, что в исследовании MOSAIC инфузия лейковорина и фторурацила определила показатели 5-ти летней выживаемости без признаков болезни в группе II стадии с факторами риска на уровне $74,6 \%$, а в группе FOLFOX - 82,3\% [52], клинически значимым могла быть признана верхняя граница 95\% доверительного интервала к отношению риска прогрессирования на уровне 1,2 для доказательства неменьшей эффективности 3 месяцев терапии 6 месяцам терапии комбинацией оксалиплатина и фторпиримидинов. Это определяет, возможность снижения показателя 5-летней выживаемости без признаков болезни лишь на 3,1\% в абсолютных значениях, то есть в группе 6 месяцев ожидаемая выживаемость составит $82,3 \%$, а в группе 3 месяцев - 79,2\%. При показателях $\alpha=0,1$ и $\beta=0,2$, необходимо было зарегистрировать как минимум 542 события прогрессирования или смерти. В качестве факторов неблагоприятного прогноза расценивались: Т4, количество изученных лимфоузлов менее 10-12, операция на фоне непроходимости или перфорации, периневральная, венозная или лимфоваскулярная инвазия, низкая степень дифференцировки. Объединение данных 4 исследований (HORG, ACHIEVE2, SCOT, TOSCA) в рамках проекта IDEA позволил включить в анализ 3273 пациента со II стадией с факторами риска: 619 больным проведена терапия 3 месяцев FOLFOX; 1020 - 3 месяца CAPOX; 635 - 6 месяцев FOLFOX; 999 - 6 месяцев САРОХ. Зарегистрировано 553 события. В группе 3 месяцев терапии частота развития нежелательных явлений 3-5 степени составила $26 \%$, а в группе 6 месяцев - $40 \%$. На всей группе пациентов не показана неменьшая эффективность 3 месяцев терапии 6 месяцам терапии комбинацией оксалиплатина и фторпиримидинов: ОР 1,17, 95\% ДИ 1,05-1,31 (должно быть не более 1,2), $\mathrm{p}$ (для неменьшей эффективности) $=0,3851$ (5-летняя выживаемость без признаков болезни составила 80,7\% против 83,9\%, соответственно). При этом не удалось найти клинических факторов, ассоциированых с неменьшей эффективностью 3 месяцев терапии - независимо от показателя Т (Т4 против Т3), ни для числа изученных лимфоузлов. Однако, при разделении пациентов на группы в зависимости от химиотерапевтического режима (FOLFOX или CAPOX), отмечено, что среди пациентов, которым проводилось лечение по схеме САРОХ, 3 месяца терапии были не менее эффективным подходом 6 месяцам лечения: 5-летняя выживаемость без признаков болезни составила $81,7 \%$ против $82 \%$, соответственно. В группе же пациентов, которым проводилась химиотерапия по схеме FOLFOX, данный показатель составил 79,2\% против 86,5\%, соответственно [53].

Таким образом, хотя добавление оксалиплатина к фторпиримидинам не приводило к значимому улучшению показателей общей выживаемости при терапии в течение 6 месяцев. Тем не менее, возможно рекомендовать применение не только 6 месяцев монотерапии фторпиримидинами, но и 3-х месяцев терапии режимом САРОХ при II стадии рака толстой кишки с факторами риска. Тем более, что токсичность 3-4 степени при 3-х месяцах терапии режимом XELOX составила всего 26\% [53], а при 6 месяцах терапии капецитабином - более 25\%, при 6 месяцах терапии режимом Меуо - более $40 \%$ (по результатам исследования Х-АСТ) [54], при комбинации de Gramont - 11,1\% [55].

Удаление первичной опухоли при метастатическом раке толстой кишки

Не будем перечислять все ретроспективные работы, которые проводились для оценки необходимости удаления первичной опухоли при метастатическом раке толстой кишки. Большинство из них четко указывало на значимое улучшение общей выживаемости при удалении первичного очага [56]. Однако в последнее время все больше исследований приносило противоречивые результаты. Так в исследовании Alawadi с соавторами, проведенный анализ 15154 пациентов, которым в 57\% наблюдений было выполнено удаление первичной опухоли, первоначально показано значимое улучшение общей выживаемости при комбинации химиотерапии с хирургией первичной опухоли (Cox model и prospensity scores - HR 0,4 и HR 0,46 , соответственно). Однако применение Landmark анализа с инструментальным анализом переменных определили отсутствие эффекта от удаления первичной опухоли в отношении общей выживаемости (ОР 0,97, 95\% ДИ 0,87-1,06) [57]. А примеры метастатического рака желудка, молочной железы и почки [58-60], требовало дождаться рандомизированных проспективных исследований посвященных данному вопросу и при раке толстой кишки. Результаты первой такой работы были представлены на симпозиуме ASCO GI 2020. Исследователи планировали рандомизировать 280 пациентов (статистическая гипотеза была пересчитана в связи с медленным набором) с бессимптомной первичной опухолью сТ1-4, наличием неоперабельных метастазов 1-3 зон, возрастом 20-74 года, отсутствием метастазов в костях и головном мозге, отсутствием асцита, отсутствием лучевой и химиотерапии в анамнезе. То есть отобрали наиболее сохранную популяцию больных, для демонстрации 
эффективности комбинированного подхода. При этом всем пациентам проводилась одинаковая химиотерапия по схеме FOLFOX/ XELOX с бевацизумабом. Основным критерием эффективности выбрана общая выживаемость. После анализа результатов лечения 160 пациентов, независимый комитет предложил завершить исследование в связи с неэффективностью сочетания хирургии первичной опухоли и химиотерапии. При медиане наблюдения 22 месяца, в группе с удалением первичной опухоли медиана продолжительности жизни составила 25,9 месяцев против 26,7 месяцев в группе только системного лечения (ОР 1,1, 95\% Ди 0,76-1,59, p=0,69). Также не различалась медиана выживаемости без прогрессирования: 10,4 против 12,1 месяцев, соответственно (ОР 1,08, 95\% ДИ 0,77-1,5). Трое пациентов погибло в послеоперационном периоде. Также удаление первичной опухоли было ассоциировано со значимым увеличением частоты нежелательных явлений 3-4 степени - 49\% против 36\%, соответственно. Ни в одном из поданализов не удалось выявить преимущества от удаления первичной опухоли. У 13 пациентов потребовалось выполнение хирургических манипуляций при осложнении течения первичной опухоли в процессе химиотерапии [61]. Конечно, можно дискутировать о высокой послеоперационной летальности, азиатской популяции больных, о совершенствовании хирургических методик, необходимости дождаться результатов еще 4 рандомизированных исследований, которые посвящены данному вопросу, но для клинической практики уже сейчас можно четко заявить, что стремиться удалять бессимптомную первичную опухоль при неоперабельных метастазах рака толстой кишки у каждого больного не нужно.

\section{HIPEC}

Еще одно знаковое исследование, представленное на конференциях в 2019 году, было посвящено роли внутрибрюшинной гипертермической химиоперфузии при раке толстой кишки. Если рассмотреть историю вопроса, следует отметить, что у 4-13\% больных раком толстой кишки выявляется поражение брюшины [62]. При этом только у 4\% - отмечается ее изолированный перитонеальный канцероматоз. Чаще метастазы по брюшине наблюдают среди пациентов с показателем T4, при перфорации стенки кишки, при поражении регионарных лимфоузлов, при низкой степени дифференцировки опухоли, муцинозном гистотипе, молодом возрасте пациентов [63]. Как и при раке желудка при изолированном поражении брюшины применение циторедукции с внутрибрюшинной химиотерапией позволяет по ряду работ достичь хороших результатов выживаемости. К примеру, анализ лечения 523 больных с изолированным поражением брюшины при раке толстой кишки привел к показателям 3-годичной общей выживаемости на уровне 41\%, а 5 летней выживаемости на уровне
27\% [64]. Было проведено даже небольшое проспективное рандомизированное исследование, показавшее преимущество комбинированного подхода над химиотерапией, только следует отметить, что в группе контроля пациенты получали только лейковорин и 5-фторурацил [65].

В 2018 году были представлены результаты первого рандомизированного исследования III фазы по определению роли внутрибрюшинной гипертермической химиоперфузии при изолированном поражении брюшины метастазами колоректального рака. Пациентам, в отсутствии экстраперитонеального поражения, при стабилизации заболевания, при индексе перитонеального канцероматоза менее 25 , предшествующей системной терапии не менее 6 месяцев, в возрасте от 18 до 70 лет, выполнялась макроскопически полная циторедукция R0/R1 или оставалась резидуальные очаги размерами $\leq 1$ мм (R2). В дальнейшем проводилась внутрибрюшинная гипертермическая химиоперфузия или все ограничивалось только циторедуктивной операцией. В качестве химиотерапии применялись: оксалиплатин - 40 мг $/ \mathrm{M}^{2}$ в течение 30 минут внтрибрюшинно и лейковорин $20 \mathrm{Mг} / \mathrm{M}^{2}$ или 5-фторурацил $400 \mathrm{мг} / \mathrm{M}^{2}$ в/в во время внутрибрюшинной процедуры. Авторы выявили отсутствие уменьшения риска прогрессирования (ОР $0,9,95 \%$ ДИ 0,69-1,19, $\mathrm{p}=0,486)$ и смерти (OР 1,0, 95\% ДИ 0,73-1,37, $\mathrm{p}=0,995$ ) при выполнении внутрибрюшинной химиотерапии. Отметим, что хотя внутрибрюшинный компонент при раке толстой кишки не работал, исследователи показали высокие показатели медианы продолжительности жизни при выполнении циторедукции при изолированном поражении брюшины - 41 месяц в обеих группах [66].

Несмотря на отрицательные результаты представленной выше работы, большинство хирургов, занимающихся внутрибрюшинной химиотерапией, ожидали еще результаты другого проспективного рандомизированного исследования, которое было представлено на конференции ASCO GI 2019. В данное исследование включались больные раком толстой кишки с высоким риском развития метастазов по брюшине (T4N0-2M0 или перфорация стенки кишки). После проведения хирургического лечения, пациентов рандомизировали на 2 группы: которым выполнялась или не выполнялась внутрибрюшинная гипертермическая химиоперфузия. В обеих группах проводилась адъювантная системная химиотерапия. Режим внутрибрюшинной химиотерапии был аналогичен предыдущей работе. В качестве основного критерия эффективности выбрана частота развития метастазов по брюшине. Для чего через 18 месяцев с момента хирургического лечения выполнялась диагностическая лапароскопия. Статистическая гипотеза предполагала, что в группе внутрибрюшинной химиотерапии уменьшится частота перитонеального канцероматоза с $25 \%$ до $10 \%$ при $\alpha 0,05$ и $\beta$ 0,2. Всего 
было рандомизировано 204 пациента. Авторы работы пришли к выводу, что ни частота развития метастазов по брюшине, ни выживаемость до развития метастазов по брюшине (ОР 0,86, 95\% ДИ 0,51-1,54) не различалась между группами сравнения. Тем не менее, следует отметить, что в течение 23 месяцев наблюдения у $21 \%$ больных развились метастазы по брюшине, что подчеркивает необходимость продолжения исследований в данной популяции больных раком толстой кишки [67].

\section{Myтащия в гене BRAF}

Субтипирование рака толстой кишки позволило выделить отдельный генетический вариант опухоли с мутацией в гене BRAF (преимущественно V600E), частота которого варьирует от 4\% до 8\%. Данный вариант опухолей ассоциирован с агрессивным течением заболевания и неблагоприятным прогнозом, низкой эффективностью стандартной химиотерапии и отсутствием эффекта от добавления анти-EGFR антител к режимам FOLFOX и FOLFIRI [68-70]. И если для первой линии терапии данного типа рака толстой кишки достигли консенсуса по применению режима FOLFOXIRI с бевацизумабом, то для второй и последующих линий эффективного лечения не было. Тем более что монотерапия ингибиторами BRAF также разочаровала отсутствием значимых достижений [71], а комбинации BRAF и MEK ингибиторов в сочетании с анти-EGFR антителами показали обнадеживающие результаты, но были ограничены исследованиями I-II фазы [72]. Только в 2019 году были представлены результаты рандомизированного исследования III фазы по сравнению комбинации BRAF ингибитора (энкорафениба), анти-EGFR антитела (цетуксимаба) с или без МЕК ингибитора (бимниметиниба) и комбинации иринотекана или режима FOLFIRI с цетуксимабом во
2-3 линии лечения больных метастатическим раком толстой кишки с мутацией в гене BRAF. В исследование было включено 605 пациентов. Основным критерием эффективности явилась общая выживаемость. Применение тройной или двойной комбинации таргетными препаратами привело к увеличению в 10 раз частоты объективных эффектов (26\% и $20 \%$, соответственно) в сравнении с контрольной группой (2\%). При этом наибольшая частота объективных эффектов зарегистрирована при применении тройной комбинации во второй линии терапии - 34\%. Применение комбинации BRAF, MEK ингибитора и анти-EGFR антитела также привело к снижению риска смерти на 48\% (ОР 52\%, 95\% ДИ 0,39-0,7, p<0,0001), что вылилось и в увеличение медианы продолжительности жизни до 9 месяцев, в сравнении с 5,4 месяцами в группе контроля [73].

В настоящий момент ни энкорафениб, ни биниметиниб, не зарегистрированы на территории РФ, тем не менее, в рекомендации АОР внесены альтернативные комбинации BRAF и MEK ингибиторов, что поможет уже сейчас назначать данной группе больных наиболее эффективное лечение во 2 и последующих линиях лечения.

В заключение необходимо подчеркнуть, что, если бы эта статья вышла до 2019 года, то она имела бы исключительно теоретическое значение, так как какие бы значимые результаты не были получены в рандомизированных исследованиях, их воплощение в практику возможно только при наличии свободного доступа к современной терапии, чего не было до последнего времени. И главным достижением в 2019 году в онкологии в России все-таки следует считать наконец-то появившуюся возможность для пациентов с онкопатологией получать, а онкологам назначать, современные препараты системной терапии.

\section{Список литературы}

1. Al-Batran S.E., Homann N., Pauligk C. et al. Perioperative chemotherapy with fluorouracil plus leucovorin, oxaliplatin, and docetaxel versus fluorouracil or capecitabine plus cisplatin and epirubicin for locally advanced, resectable gastric or gastro-oesophageal junction adenocarcinoma (FLOT4): a randomised, phase $2 / 3$ trial // Lancet. - 2019 May 11. - Vol. 393, № 10184. - P. 1948-1957.

2. Sakuramoto S., Sasako M., Yamaguchi T. et al. Adjuvant chemotherapy for gastric cancer with S-1, an oral fluoropyrimidines // N Engl J Med. - 2007 Nov 1. - Vol. 357, № 18. - P. 1810-20.

3. Sasako M., Sakuramoto S., Katai H. et al. Five-year outcomes of a randomized phase III trial comparing adjuvant chemotherapy with S-1 versus surgery alone in stage II or III gastric cancer.https://www.ncbi.nlm.nih.gov/ pubmed/22010012 - 2011 Nov 20. - Vol. 29, № 33. - P. 4387-93.

4. Bang Y.J., Kim Y.W., Yang H.K. et al. Adjuvant capecitabine and oxaliplatin for gastric cancer after D2 gastrectomy (CLASSIC): a phase 3 open-label, randomised controlled trial // Lancet. - 2012 Jan 28. - Vol. 379, № 9813. - P. 315-21.

5. Nob S.H., Park S.R., Yang H.K. et al. Adjuvant capecitabine plus oxaliplatin for gastric cancer after D2 gastrectomy (CLASSIC): 5-year follow-up of an open-label, randomised phase 3 trial // Lancet Oncol. - 2014 Nov. - Vol. 15, № 12. - P. 1389-96. 
6. Park S.H., Zang D.Y., Han B. et al. ARTIST 2: Interim results of a phase III trial involving adjuvant chemotherapy and/or chemoradiotherapy after D2-gastrectomy in stage II/III gastric cancer (GC) // J Clin Oncol. - 2019. - Vol. 37 (suppl; abstr 4001).

7. Kang Y., Yook J.H., Park Y. et al. Phase III randomized study of neoadjuvant chemotherapy (CT) with docetaxel (D), oxaliplatin (O) and S-1 (S) (DOS) followed by surgery and adjuvant S-1, vs surgery and adjuvant S-1, for resectable advanced gastric cancer (GC) (PRODIGY) // Annals of Oncology. -2019. - Vol. 30 (suppl_5) - P. v851-v934.

8. Wang X., Li S., Xie T. et al. Early results of the randomized, multicenter, controlled evaluation of S-1 and oxaliplatin as neoadjuvant chemotherapy for Chinese advanced gastric cancer patients (RESONANCE Trial) // J Clin Oncol. 2020. - Vol. 38 (suppl 4; abstr 280).

9.JiJ., Shen L., LiZ. et al. Perioperative Chemotherapy of Oxaliplatin Combined with S-1 (SOX) versus Postoperative Chemotherapy of SOX or Oxaliplatin with Capecitabine (XELOX) in Locally Advanced Gastric Adenocarcinoma with D2 Gastrectomy: a Randomized Phase III Trial (RESOLVE Trial) // Annals of Oncology. - 2019. - Vol. 30 (suppl_5). P. v851-v934.

10. Hall P.S., Swinson D., Waters J.S. et al. Optimizing chemotherapy for frail and elderly patients (pts) with advanced gastroesophageal cancer (aGOAC): The GO2 phase III trial // J Clin Oncol. - 2019. - Vol. 37 (suppl; abstr 4006).

11. Hall P.S., Lord S.R., Collinson M., et al. A randomised phase II trial and feasibility study of palliative chemotherapy in frail or elderly patients with advanced gastroesophageal cancer (321GO) // Br J Cancer. - 2017 Feb 14. - Vol. 116, № 4. - P. 472-478.

12.XuR., Wang Z.Q., Shen L. et al. S-1 plus oxaliplatin versus S-1 plus cisplatin as first-line treatment for advanced diffuse-type or mixed-type gastric/gastroesophageal junction adenocarcinoma: A randomized, phase 3 trial // J Clin Oncol. - 2019. - Vol. 37 (suppl; abstr 017).

13. Cunningham D., Starling N., Rao S. et al. Capecitabine and oxaliplatin for advanced esophagogastric cancerhttps://www.ncbi.nlm.nih.gov/pubmed/18172173 - 2008 Jan 3. - Vol. 58, № 1. - P. 36-46.

14. Yamada Y., Boku N., Mizusawa J. et al. Phase III study comparing triplet chemotherapy with S-1 and cisplatin plus docetaxel versus doublet chemotherapy with S- 1 and cisplatin for advanced gastric cancer (JCOG1013) // Journal of Clinical Oncology. - 2018. - Vol. 36 (№ 15 suppl). - P. 4009-4009.

15. Al-Batran S.E., Pauligk C., Homann N. et al. The feasibility of triple-drug chemotherapy combination in older adult patients with oesophagogastric cancer: a randomised trial of the Arbeitsgemeinschaft Internistische Onkologie (FLOT65+) // Eur J Cancer. - 2013 Mar. - Vol. 49, № 4. - P. 835-42.

16. Lin R., Chen Y., Zhu J. et al. POF (paclitaxel plus FOLFOX) versus IP PAC (intraperitoneal paclitaxel plus FOLFOX) versus FOLFOX as a first-line treatment in advanced gastric cancer (AGC): Update from a multicenter, randomized phase II trial, FNF-004 trial // Journal of Clinical Oncology. - 2019. - Vol. 37 (№ 15_suppl). - P. 4035-4035.

17. Li J., Qin S., Xu J. et al. Randomized, Double-Blind, Placebo-Controlled Phase III Trial of Apatinib in Patients With Chemotherapy-Refractory Advanced or Metastatic Adenocarcinoma of the Stomach or Gastroesophageal Junction // J Clin Oncol. - 2016 May 1. - Vol. 34, № 13. - P. 1448-54.

18. Kang Y., Kang W.K., Di Bartolomeo M. et al. Randomized phase 3 ANGEL study of rivoceranib (apatinib) + best supportive care (BSC) vs placebo + BSC in patients with advanced/ metastatic gastric cancer who failed 2 prior chemotherapy regimens // Annals of Oncology. - 2019. - Vol. 30 (suppl_5). - P. v851-v934.

19. Kang Y.K., Boku N., Satob T. et al. Nivolumab in patients with advanced gastric or gastro-oesophageal junction cancer refractory to, or intolerant of, at least two previous chemotherapy regimens (ONO-4538-12, ATTRACTION-2): a randomised, double-blind, placebo-controlled, phase 3 trial // Lancet. - 2017 Dec 2. - Vol. 390, № 10111. - P. 2461-2471.

20. Bang Y.J., Ruiz E.Y., Van Cutsem E. et al. Phase III, randomised trial of avelumab versus physician's choice of chemotherapy as third-line treatment of patients with advanced gastric or gastro-oesophageal junction cancer: primary analysis of JAVELIN Gastric 300 // Ann Oncol. - 2018 Oct 1. - Vol. 29, № 10. - P. 2052-2060.

21. Shitara K., Özgüroğlu M., Bang Y.J. et al. Pembrolizumab versus paclitaxel for previously treated, advanced gastric or gastro-oesophageal junction cancer (KEYNOTE-061): a randomised, open-label, controlled, phase 3 trial // Lancet. 2018 Jul 14. - Vol. 392, № 10142. - P. 123-133.

22. Bang Y.J., Cho J.Y., Kim Y.H. et al. Efficacy of sequential ipilimumab monotherapy versus best supportive care for unresectable locally advanced/metastatic gastric or gastroesophageal junction cancer // Clin Cancer Res. - 2017 Oct 1. - Vol. 23, № 19. - P. 5671-5678.

23. Kim S.B., Doi .T, Kato K. et al. 124O-KEYNOTE-181: Pembrolizumab vs chemotherapy in patients (pts) with advanced/metastatic adenocarcinoma (AC) or squamous cell carcinoma (SCC) of the esophagus as second-line (2L) therapy // Annals of Oncology. - 2019. - Vol. 30 (Supplement 9). - P. ix42-ix67.

24. Moebler M.H., Dvorkin M., Ozguroglu M. et al. Results of the JAVELIN Gastric 100 phase 3 trial: avelumab maintenance following first-line (1L) chemotherapy (CTx) vs continuation of CTx for HER2- advanced gastric or gastroesophageal junction cancer (GC/GEJC) // J Clin Oncol. - 2020. - Vol. 38 (suppl 4; abstr 278).

25. Cunningham D., Fong C.Y.K., Peckitt C. et al. Evaluating maintenance therapies in advanced oesophago-gastric adenocarcinoma (OGA): Interim analysis and biomarker results from the PLATFORM study // J Clin Oncol. 2020. - Vol. 38 (suppl 4; abstr 282).

26. Shitara K., Van Cutsem E., Bang Y. et al. Pembrolizumab With or Without Chemotherapy vs Chemotherapy in Patients With Advanced G/ GEJ Cancer (GC) Including Outcomes According to Microsatellite Instability-High (MSI-H) Status in KEYNOTE-062 // Annals of Oncology. - 2019. - 30 (suppl_5). - P. v851-v934. 
27. Shitara K., Özgüroğlu M., Bang Y.J. et al. KEYNOTE-061: Phase 3 study of pembrolizumab vs paclitaxel for previously treated advanced gastric or gastroesophageal junction (G/GEJ) cancer // Annals of oncology. - 2018 June. - Vol. 29., Supplement 5. - P. v123.

28. Diaz L.A., Le D., Maio M. et al. Pembrolizumab in microsatellite instability high cancers: updated analysis of the phase 2 KEYNOTE-164 and KEYNOTE-158 studies // Annals of Oncology. - 2019. - Vol. 30 (suppl_5). - P. v475-v532.

29. El-Khoueiry A.B., Sangro B., Yau T. et al. Nivolumab in patients with advanced hepatocellular carcinoma (CheckMate 040): an open-label, non-comparative, phase 1/2 dose escalation and expansion trial // Lancet. - 2017 Jun 24. - Vol. 389, № 10088. - P. 2492-2502.

30.Zbu A.X., Finn R.S., Edeline J. et al. Pembrolizumab in patients with advanced hepatocellular carcinoma previously treated with sorafenib (KEYNOTE-224): a non-randomised, open-label phase 2 trial // Lancet Oncol. - 2018 Jul. Vol. 19, № 7. - P. 940-952.

31. Finn R.S., Ryoo B.Y., Merle P. et al. Pembrolizumab As Second-Line Therapy in Patients With Advanced Hepatocellular Carcinoma in KEYNOTE-240: A Randomized, Double-Blind, Phase III Trial // J Clin Oncol. - 2020 Jan 20. - Vol. 38, № 3. - P. 193-202.

32. Cheng A., Qin S., Ikeda M. et al. IMbrave150: Efficacy and safety results from a ph III study evaluating atezolizumab (atezo) + bevacizumab (bev) vs sorafenib (Sor) as first treatment (tx) for patients (pts) with unresectable hepatocellular carcinoma (HCC) // Annals of Oncology. - 2019. - Vol. 30 (suppl_9). - P. ix183-ix202.

33. Galle P.R., Finn R.S., Qin S. et al. Patient-reported outcomes (PROs) from the Phase III IMbrave150 trial of atezolizumab (atezo) + bevacizumab (bev) vs sorafenib (sor) as first-line treatment (tx) for patients (pts) with unresectable hepatocellular carcinoma (HCC) // J Clin Oncol. - 2020. - Vol. 38 (suppl 4; abstr 476).

34. Conroy T., Hammel P., Hebbar M. et al. FOLFIRINOX or Gemcitabine as Adjuvant Therapy for Pancreatic Cancer // N Engl J Med. - 2018 Dec 20. - Vol. 379, № 25. - P. 2395-2406.

35. Neoptolemos J., Palmer D., Ghaneb P. et al. Comparison of adjuvant gemcitabine and capecitabine with gemcitabine monotherapy in patients with resected pancreatic cancer (ESPAC-4): a multicentre, open-label, randomised, phase 3 trial // Lancet. - 2017 Mar 11. - Vol. 389, № 10073. - P. 1011-1024.

36. Neoptolemos J.P., Dunn J.A., Stocken D.D. et al. Adjuvant chemoradiotherapy and chemotherapy in resectable pancreatic cancer: a randomised controlled trial // Lancet. - 2001. - Vol. 358 - P. 1576-85.

37. Neoptolemos J.P., Stocken D.D., Friess H. et al. A randomized trial of chemoradiotherapy and chemotherapy after resection of pancreatic cancer // N Engl J Med. - 2004. - Vol. 350 - P. 1200-10.

38. Oettle H., Post S., Neubaus P. et al. Adjuvant chemotherapy with gemcitabine vs observation in patients undergoing curative-intent resection of pancreatic cancer: a randomized controlled trial // JAMA. - 2007. Vol. 297 - P. 267-77.

39. Tempero M.A., Reni M., Riess H. et al. APACT: phase III, multicenter, international, open-label, randomized trial of adjuvant nab-paclitaxel plus gemcitabine (nab-P/G) vs gemcitabine $(G)$ for surgically resected pancreatic adenocarcinoma // Journal of Clinical Oncology. - 2019. - Vol. 37, № 15_suppl. - P. 4000-4000.

40. Conroy T., Desseigne F., Ychou M. et al. FOLFIRINOX versus gemcitabine for metastatic pancreatic cancer // N Engl J Med. - 2011. - Vol. 364, № 19 - P. 1817-25.

41.Von Hoff D., Ervin T., Arena F. et al. Increased Survival in Pancreatic Cancer with nab-Paclitaxel plus Gemcitabine // N Engl J Med. - 2013. - Vol. 369. - P. 1691-703.

42. Dahan L., Phelip J.M., Le Malicot K. et al. FOLFIRINOX until progression, FOLFIRINOX with maintenance treatment, or sequential treatment with gemcitabine and FOLFIRI.3 for first-line treatment of metastatic pancreatic cancer: A randomized phase II trial (PRODIGE 35-PANOPTIMOX) // Journal of Clinical Oncology. - 2018. - Vol. 36 (№ 15_suppl). - P. 4000-4000.

43. Golan T., Hammel P., Reni M. et al. Maintenance Olaparib for Germline BRCA-Mutated Metastatic Pancreatic Cancer // N Engl J Med. - 2019 Jul 25. - Vol. 381, № 4. - P. 317-327.

44. O'Reilly E.M., Lee J.W., Zalupski M. et al. Randomized, Multicenter, Phase II Trial of Gemcitabine and Cisplatin With or Without Veliparib in Patients With Pancreas Adenocarcinoma and a Germline BRCA/ PALB2 Mutation // J Clin Oncol. - 2020 Jan 24. - JCO1902931.

45. Francois Y., Nemoz C.J., Baulieux J. et al. Influence of the interval between preoperative radiation therapy and surgery on downstaging and on the rate of sphincter-sparing surgery for rectal cancer: the Lyon R90-01 randomized trial // J Clin Oncol. - 1999 Aug. - Vol. 17, № 8. - P. 2396.

46. Probst C.P., Becerra A.Z., Aquina C.T. et al. Extended intervals after neoadjuvant therapy in locally advanced rectal cancer: the key to improved tumor response and potential organ preservation // J Am Coll Surg. - 2015 August. Vol. 221, № 2. - P. 430-440.

47. Lee S.W., Lee J.H., Lee I.K. et al. The impact of surgical timing on pathologic tumor response after short course and long course preoperative chemoradiation for locally advanced rectal adenocarcinoma // Cancer Res Treat. 2018. - Vol. 50. № 3. - P. 1039-1050.

48. OnJ., Aly E.H. Watch and wait' in rectal cancer: summary of the current evidence // Int J Colorectal Dis. - 2018 Sep. - Vol. 33, № 9. - P. 1159-1168.

49. Kwak Y.K., Kim K., Lee J.H. et al. Timely tumor response analysis after preoperative chemoradiotherapy and curative surgery in locally advanced rectal cancer: A multi-institutional study for optimal surgical timing in rectal cancer // Radiother Oncol. - 2016 Jun. - Vol. 119, № 3. - P. 512-8. 
50. Lefevre J.H., Mineur L., Cachanado M, et al. Does a longer waiting period after neoadjuvant radiochemotherapy improve the oncological prognosis of rectal cancer? Three-year follow-up results of the GRECCAR- 6 randomized multicenter trial // J Clin Oncol. - 2019. - Vol. 37 (suppl 4; abstr 483).

51. Grothey A., Sobrero A.F., Shields A.F. et al. Duration of Adjuvant Chemotherapy for Stage III Colon Cancer // N Engl J Med. - 2018. - Vol. 378. - P. 1177-1188.

52. Andre T., Boni C., Navarro M., et al. Improved overall survival with oxaliplatin, fluorouracil, and leucovorin as adjuvant treatment in stage II or III colon cancer in the MOSAIC trial // J Clin Oncol. - 2009. - Vol. 27, № 19. P. 3109-3116.

53. Iveson T., Sobrero A.F., Yoshino T. et al. Prospective pooled analysis of four randomized trials investigating duration of adjuvant (adj) oxaliplatin-based therapy (3 vs 6 months $\{\mathrm{m}\}$ ) for patients (pts) with high-risk stage II colorectal cancer (CC) // J Clin Oncol. - 2019. - Vol. 37 (suppl; abstr 3501).

54. Twelves C., Wong A., Nowacki M.P. et al. Capecitabine as adjuvant treatment for stage III colon cancer // N Engl J Med. - 2005 Jun 30. - Vol. 352, № 26. - P. 2696-704.

55. De Gramont A., Bosset J-F., Milan C. et al. Randomized trial comparing monthly low-dose leucovorin and fluorouracil bolus with bimonthly high-dose leucovorin and fluorouracil bolus plus continuous infusion for advanced colorectal cancer: French intergroup study // J Clin Oncol. - 1997. - Vol. 15 - P. 808-815.

56. Федянин М.Ю., Трякин А.А., Тюляндин С.А. Необходимо ли удаление бессимптомной первичной опухоли при нерезектабельном метастатическом раке толстой кишки? // Тазовая хирургия и онкология. - 2015. - Т. 5 , № 3. - C. 9-14.

57. Alawadi Z., Phatak U.R., Hu C.Y. et al. Comparative effectiveness of primary tumor resection in patients with stage IV colon cancer // Cancer. - 2017 Apr 1. - Vol. 123, № 7. - P. 1124-1133.

58. Fujitani K., Yang H.K., Mizusawa J. et al. Gastrectomy plus chemotherapy versus chemotherapy alone for advanced gastric cancer with a single non-curable factor (REGATTA): a phase 3, randomised controlled trial // Lancet Oncol. - 2016 Mar. - Vol. 17, № 3. - P. 309-18.

59. Tosello G., Torloni M.R., Mota B.S. et al. Breast surgery for metastatic breast cancer // Cochrane Database Syst Rev. - 2018 Mar 15. - Vol. 3. - CD011276.

60. Méjean A., RavaudA., Thezenas S. et al. Sunitinib Alone or after Nephrectomy in Metastatic Renal-Cell Carcinoma // N Engl J Med. - 2018 Aug 2. - Vol. 379, № 5. - P. 417-427.

61. Kanemitsu Y., Shitara K., Mizusawa J. et al. A randomized phase III trial comparing primary tumor resection plus chemotherapy with chemotherapy alone in incurable stage IV colorectal cancer: JCOG1007 study (iPACS) // J Clin Oncol. -2020. - Vol. 38 (suppl 4; abstr 7).

62. Koppe M.J., Boerman O.C., Oyen W.J., Bleichrodt R.P. Peritoneal carcinomatosis of colorectal origin: incidence and current treatment strategies // Ann Surg. - 2006. - Vol. 243 - P. 212-222.

63. Klaver Y.L., Lemmens V.E., Nienhuijs S.W., Luyer M.D., de Hingh I.H. Peritoneal carcinomatosis of colorectal origin: Incidence, prognosis and treatment options // World J Gastroenterol. - 2012. - Vol. 18 - P. 5489-5494.

64. Elias D., Gilly F., Boutitie F., Quenet F., Bereder J.M., Mansvelt B., Lorimier G., Dubu P., Glehen O. Peritoneal colorectal carcinomatosis treated with surgery and perioperative intraperitoneal chemotherapy: retrospective analysis of 523 patients from a multicentric French study // J Clin Oncol. - 2010. - Vol. 28. - P. 63-68.

65. Verwaal V.J., Bruin S., Boot H., van Slooten G., van Tinteren H. 8-year follow-up of randomized trial: cytoreduction and hyperthermic intraperitoneal chemotherapy versus systemic chemotherapy in patients with peritoneal carcinomatosis of colorectal cancer // Ann Surg Oncol. - 2008. - Vol. 15. - P. 2426-2432.

66. Quene F., Elias D., Roca L. et al. A UNICANCER phase III trial of hyperthermic intra-peritoneal chemotherapy (HIPEC) for colorectal peritoneal carcinomatosis (PC): PRODIGE $7 / /$ Journal of Clinical Oncology. - 2018. Vol. 36 (№ 18 suppl).

67. Klaver C.E.L., Wisselink D.D., Punt C.J.A. et al. Adjuvant HIPEC in patients with colon cancer at high risk of peritoneal metastases: Primary outcome of the COLOPEC multicenter randomized trial // J Clin Oncol. - 2019. Vol. 37 (suppl 4; abstr 482).

68. Yuan Z-X., Wang X-Y., Qin Q-Y. et al. The Prognostic Role of BRAF Mutation in Metastatic Colorectal Cancer Receiving Anti-EGFR Monoclonal Antibodies: A Meta-Analysis // PLoS ONE. - 2013. - Vol. 8, № 6. - e65995.

69. Mao C., Liao R.Y., Qiu L.X., Wang X.W., Ding H., Chen Q. BRAF V600E mutation and resistance to anti-EGFR monoclonal antibodies in patients with metastatic colorectal cancer: a meta-analysis // Molecular Biology Reports. - 2011. - Vol. 38 - P. 2219-23.

70. Qi X.U., An Tao X.U., Ming Ming Z.H.U. et al. Predictive and prognostic roles of BRAF mutation in patients with metastatic colorectal cancer treated with anti-epidermal growth factor receptor monoclonal antibodies: A metaanalysis // Journal of Digestive Diseases. - 2013. - Vol. 14. - P. 409-416.

71. Kopetz S., Desai J., Chan E. et al. PLX4032 in metastatic colorectal cancer patients with mutant BRAF tumors // J Clin Oncol. - 2010. - Vol. 28, № 15 (suppl 3534).

72. Федянин М.Ю., ТрякинА.А., Тюляндин С.А. Перспективы лечения больных раком толстой кишки с мутацией в гене BRAF // Тазовая хирургия и онкология. - 2014. - Т. 3. - С. 9-16.

73. Kopetz S., Grothey A., Yaeger R, et al. Encorafenib, binimetinib, and cetuximab in BRAF V600E-mutated colorectal cancer // N Engl J Med. - 2019 Oct 24. - Vol. 381, № 17. - P. 1632-1643. 


\section{References}

1. Al-Batran S.E., Homann N., Pauligk C. et al. Perioperative chemotherapy with fluorouracil plus leucovorin, oxaliplatin, and docetaxel versus fluorouracil or capecitabine plus cisplatin and epirubicin for locally advanced, resectable gastric or gastro-oesophageal junction adenocarcinoma (FLOT4): a randomised, phase 2/3 trial. Lancet. 2019 May 11; 393(10184): 1948-1957. doi: 10.1016/S0140-6736(18)32557-1.

2. Sakuramoto S., Sasako M., Yamaguchi T. et al. Adjuvant chemotherapy for gastric cancer with S-1, an oral fluoropyrimidine. N Engl J Med. 2007 Nov 1; 357(18): 1810-20.

3. Sasako M., Sakuramoto S., Katai H. et al. Five-year outcomes of a randomized phase III trial comparing adjuvant chemotherapy with S-1 versus surgery alone in stage II or III gastric cancer. J Clin Oncol. 2011 Nov 20; 29(33): 438793. doi: 10.1200/JCO.2011.36.5908.

4. Bang Y.J., Kim Y.W., Yang H.K. et al. Adjuvant capecitabine and oxaliplatin for gastric cancer after D2 gastrectomy (CLASSIC): a phase 3 open-label, randomised controlled trial. Lancet. 2012 Jan 28; 379(9813): 315-21. doi: 10.1016/ S0140-6736(11)61873-4.

5. Nob S.H., Park S.R., Yang H.K. et al. Adjuvant capecitabine plus oxaliplatin for gastric cancer after D2 gastrectomy (CLASSIC): 5-year follow-up of an open-label, randomised phase 3 trial. Lancet Oncol. 2014 Nov; 15(12): 1389-96. doi: $10.1016 / S 1470-2045(14) 70473-5$.

6. Park S.H., Zang D.Y., Han B. et al. ARTIST 2: Interim results of a phase III trial involving adjuvant chemotherapy and/or chemoradiotherapy after D2-gastrectomy in stage II/III gastric cancer (GC). J Clin Oncol. 2019; 37 (suppl; abstr 4001). doi: 10.1200/JCO.2019.37.15_suppl.4001.

7. Kang Y., Yook J.H., Park Y. et al. Phasé III randomized study of neoadjuvant chemotherapy (CT) with docetaxel (D), oxaliplatin (O) and S-1 (S) (DOS) followed by surgery and adjuvant S-1, vs surgery and adjuvant S-1, for resectable advanced gastric cancer (GC) (PRODIGY). Annals of Oncology. 2019; 30 (suppl_5): v851-v934. doi: 10.1093/annonc/ mdz394.

8. Wang X., Li S., Xie T. et al. Early results of the randomized, multicenter, controlled evaluation of S-1 and oxaliplatin as neoadjuvant chemotherapy for Chinese advanced gastric cancer patients (RESONANCE Trial). J Clin Oncol. 2020; 38 (suppl 4; abstr 280).

9.JiJ., Shen L., LiZ. et al. Perioperative Chemotherapy of Oxaliplatin Combined with S-1 (SOX) versus Postoperative Chemotherapy of SOX or Oxaliplatin with Capecitabine (XELOX) in Locally Advanced Gastric Adenocarcinoma with D2 Gastrectomy: a Randomized Phase III Trial (RESOLVE Trial). Annals of Oncology. 2019; 30 (suppl_5): v851-v934. doi: $10.1093 /$ annonc/mdz394.

10. Hall P.S., Swinson D., WatersJ.S. et al. Optimizing chemotherapy for frail and elderly patients (pts) with advanced gastroesophageal cancer (aGOAC): The GO2 phase III trial. J Clin Oncol. 2019; 37 (suppl; abstr 4006). doi: 10.1200/ JCO.2019.37.15_suppl.4006.

11. Hall P.S., Lord S.R., Collinson M. et al. A randomised phase II trial and feasibility study of palliative chemotherapy in frail or elderly patients with advanced gastroesophageal cancer (321GO). Br J Cancer. 2017 Feb 14; 116(4): 472478. doi: 10.1038/bjc.2016.442.

12. Xu R., Wang Z.Q., Shen L. et al. S-1 plus oxaliplatin versus S-1 plus cisplatin as first-line treatment for advanced diffuse-type or mixed-type gastric/ gastroesophageal junction adenocarcinoma: A randomized, phase 3 trial. J Clin Oncol. 2019; 37 (suppl; abstr. 017). doi: 10.1200/JCO.2019.37.15 suppl.4017.

13. Cunningham D., Starling N., Rao S. et al. Capecitabine and oxaliplatin for advanced esophagogastric cancer. N Engl J Med. 2008 Jan 3; 358(1): 36-46. doi: 10.1056/NEJMoa073149.

14. Yamada Y., Boku N., Mizusawa J. et al. Phase III study comparing triplet chemotherapy with S-1 and cisplatin plus docetaxel versus doublet chemotherapy with S-1 and cisplatin for advanced gastric cancer (JCOG1013). Journal of Clinical Oncology. 2018; 36 (no. 15 suppl): 4009-4009. doi: 10.1200/JCO.2018.36.15 suppl.4009.

15. Al-Batran S.E., Pauligk C., Homann N. et al. The feasibility of triple-drug chemotherapy combination in older adult patients with oesophagogastric cancer: a randomised trial of the Arbeitsgemeinschaft Internistische Onkologie (FLOT65+). Eur J Cancer. 2013 Mar; 49(4): 835-42. doi: 10.1016/j.ejca.2012.09.025.

16. Lin R., Chen Y., Zhu J. et al. POF (paclitaxel plus FOLFOX) versus IP PAC (intraperitoneal paclitaxel plus FOLFOX) versus FOLFOX as a first-line treatment in advanced gastric cancer (AGC): Update from a multicenter, randomized phase II trial, FNF-004 trial. Journal of Clinical Oncology. 2019; 37 (no. 15_suppl.): 4035-4035. doi: 10.1200/JCO.2019.37.15_suppl.4035.

17.LiJ., Qin S., Xu J. et al. Randomized, Double-Blind, Placebo-Controlled Phase III Trial of Apatinib in Patients With Chemotherapy-Refractory Advanced or Metastatic Adenocarcinoma of the Stomach or Gastroesophageal Junction. J Clin Oncol. 2016 May 1; 34(13): 1448-54. doi: 10.1200/JCO.2015.63.5995.

18. Kang Y., Kang W.K., Di Bartolomeo M. et al. Randomized phase 3 ANGEL study of rivoceranib (apatinib) + best supportive care (BSC) vs placebo + BSC in patients with advanced/ metastatic gastric cancer who failed 2 prior chemotherapy regimens. Annals of Oncology. 2019; 30 (suppl_5): v851-v934. doi: 10.1093/annonc/mdz394.

19. Kang Y.K., Boku N., Satob T. et al. Nivolumab in patients with advanced gastric or gastro-oesophageal junction cancer refractory to, or intolerant of, at least two previous chemotherapy regimens (ONO-4538-12, ATTRACTION-2): a randomised, double-blind, placebo-controlled, phase 3 trial. Lancet. 2017 Dec 2; 390(10111): 2461-2471. doi: $10.1016 /$ S0140-6736(17)31827-5. 
20. Bang Y.J., Ruiz E.Y., Van Cutsem E. et al. Phase III, randomised trial of avelumab versus physician's choice of chemotherapy as third-line treatment of patients with advanced gastric or gastro-oesophageal junction cancer: primary analysis of JAVELIN Gastric 300. Ann Oncol. 2018 Oct 1; 29(10): 2052-2060. doi: 10.1093/annonc/mdy264.

21. Shitara K., Özgüroğlu M., Bang Y.J. et al. Pembrolizumab versus paclitaxel for previously treated, advanced gastric or gastro-oesophageal junction cancer (KEYNOTE-061): a randomised, open-label, controlled, phase 3 trial. Lancet. 2018 Jul 14; 392(10142): 123-133. doi: 10.1016/S0140-6736(18)31257-1.

22. Bang Y.J., Cho J.Y., Kim Y.H. et al. Efficacy of sequential ipilimumab monotherapy versus best supportive care for unresectable locally advanced/metastatic gastric or gastroesophageal junction cancer. Clin Cancer Res. 2017 Oct 1; 23(19): 5671-5678. doi: 10.1158/1078-0432.CCR-17-0025.

23. Kim S.B., Doi .T, Kato K. et al. $124 \mathrm{O}$ - KEYNOTE-181: Pembrolizumab vs chemotherapy in patients (pts) with advanced/metastatic adenocarcinoma (AC) or squamous cell carcinoma (SCC) of the esophagus as second-line (2L) therapy. Annals of Oncology. 2019; 30 (Supplement 9): ix42-ix67. doi: 10.1093/annonc/mdz422.

24. Moebler M.H., Dvorkin M., Ozguroglu M. et al. Results of the JAVELIN Gastric 100 phase 3 trial: avelumab maintenance following first-line (1L) chemotherapy (CTx) vs continuation of CTx for HER2- advanced gastric or gastroesophageal junction cancer (GC/ GEJC). J Clin Oncol. 202; 38 (suppl 4; abstr. 278).

25. Cunningham D., Fong C.Y.K., Peckitt C. et al. Evaluating maintenance therapies in advanced oesophago-gastric adenocarcinoma (OGA): Interim analysis and biomarker results from the PLATFORM study. J Clin Oncol. 2020; 38 (suppl 4; abstr. 282).

26. Shitara K., Van Cutsem E., Bang Y, et al. Pembrolizumab With or Without Chemotherapy vs Chemotherapy in Patients With Advanced G/ GEJ Cancer (GC) Including Outcomes According to Microsatellite Instability-High (MSI-H) Status in KEYNOTE-062. Annals of Oncology. 2019; 30 (suppl 5): v851-v934. doi: 10.1093/annonc/mdz394.

27. Shitara K., Özgüroğlu M., Bang Y.J. et al. KEYNOTE-061: Phase 3 study of pembrolizumab vs paclitaxel for previously treated advanced gastric or gastroesophageal junction (G/ GEJ) cancer. Annals of oncology. 2018; 29, Suppl.t 5: v123. doi: 10.1093/annonc/mdy208.

28. Diaz L.A., Le D., Maio M. et al. Pembrolizumab in microsatellite instability high cancers: updated analysis of the phase 2 KEYNOTE-164 and KEYNOTE-158 studies. Annals of Oncology. 2019; 30 (suppl_5): v475-v532. doi: 10.1093/ annonc/mdz253.

29. El-Khoueiry A.B., Sangro B., Yau T. et al. Nivolumab in patients with advanced hepatocellular carcinoma (CheckMate 040): an open-label, non-comparative, phase 1/2 dose escalation and expansion trial. Lancet. 2017 Jun 24; 389(10088): 2492-2502. doi: 10.1016/S0140-6736(17)31046-2.

30.Zbu A.X., Finn R.S., Edeline J. et al. Pembrolizumab in patients with advanced hepatocellular carcinoma previously treated with sorafenib (KEYNOTE-224): a non-randomised, open-label phase 2 trial. Lancet Oncol. 2018 Jul; 19(7): 940-952. doi: 10.1016/S1470-2045(18)30351-6.

31. Finn R.S., Ryoo B.Y., Merle P. et al. Pembrolizumab As Second-Line Therapy in Patients With Advanced Hepatocellular Carcinoma in KEYNOTE-240: A Randomized, Double-Blind, Phase III Trial. J Clin Oncol. 2020 Jan 20; 38(3): 193-202. doi: 10.1200/JCO.19.01307.

32. Cheng A., Qin S., Ikeda M. et al. IMbrave150: Efficacy and safety results from a ph III study evaluating atezolizumab (atezo) + bevacizumab (bev) vs sorafenib (Sor) as first treatment (tx) for patients (pts) with unresectable hepatocellular carcinoma (HCC). Annals of Oncology. 2019; 30 (suppl_9): x183-ix202. doi: 10.1093/annonc/mdz446.

33. Galle P.R., Finn R.S., Qin S. et al. Patient-reported outcomes (PROs) from the Phase III IMbrave150 trial of atezolizumab (atezo) + bevacizumab (bev) vs sorafenib (sor) as first-line treatment (tx) for patients (pts) with unresectable hepatocellular carcinoma (HCC). J Clin Oncol. 2020; 38 (suppl 4; abstr 476).

34. Conroy T., Hammel P., Hebbar M. et al. FOLFIRINOX or Gemcitabine as Adjuvant Therapy for Pancreatic Cancer. N Engl J Med. 2018 Dec 20; 379(25): 2395-2406. doi: 10.1056/NEJMoa1809775.

35. Neoptolemos J., Palmer D., Ghaneh P. et al. Comparison of adjuvant gemcitabine and capecitabine with gemcitabine monotherapy in patients with resected pancreatic cancer (ESPAC-4): a multicentre, open-label, randomised, phase 3 trial. Lancet. 2017 Mar 11; 389(10073): 1011-1024. doi: 10.1016/S0140-6736(16)32409-6.

36. Neoptolemos J.P., Dunn J.A., Stocken D.D. et al. Adjuvant chemoradiotherapy and chemotherapy in resectable pancreatic cancer: a randomised controlled trial. Lancet. 2001; 358: 1576-85. doi: 10.1016/S0140-6736(01)06651-X.

37. Neoptolemos J.P., Stocken D.D., Friess H. et al. A randomized trial of chemoradiotherapy and chemotherapy after resection of pancreatic cancer. N Engl J Med. 2004; 350: 1200-10. doi: 10.1056/NEJMoa032295.

38. Oettle H., Post S., Neubaus P. et al. Adjuvant chemotherapy with gemcitabine vs observation in patients undergoing curative-intent resection of pancreatic cancer: a randomized controlled trial. JAMA. 2007; 297: 267-77. doi: $10.1001 /$ jama.297.3.267.

39. Tempero M.A., Reni M., Riess H. et al. APACT: phase III, multicenter, international, open-label, randomized trial of adjuvant nab-paclitaxel plus gemcitabine (nab-P/G) vs gemcitabine $(G)$ for surgically resected pancreatic adenocarcinoma. Journal of Clinical Oncology. 2019; 37 (no. 15_suppl): 4000-4000. doi: 10.1200/JCO.2019.37.15_ suppl.4000.

40. Conroy T., Desseigne F., Ychou M. et al. FOLFIRINOX versus gemcitabine for metastatic pancreatic cancer. N Engl J Med. 2011; 364(19): 1817-25.

41.Von HoffD., Ervin T., Arena F. et al. Increased Survival in Pancreatic Cancer with nab-Paclitaxel plus Gemcitabine. N Engl J Med. 2013; 369: 1691-703. 
42. Dahan L., Phelip J.M., Le Malicot K. et al. FOLFIRINOX until progression, FOLFIRINOX with maintenance treatment, or sequential treatment with gemcitabine and FOLFIRI.3 for first-line treatment of metastatic pancreatic cancer: A randomized phase II trial (PRODIGE 35-PANOPTIMOX). Journal of Clinical Oncology. 2018; 36, no. 15_suppl: 4000-4000. doi: 10.1200/JCO.2018.36.15_suppl.4000.

43. Golan T., Hammel P., Reni M. et al. Maintenance Olaparib for Germline BRCA-Mutated Metastatic Pancreatic Cancer. N Engl J Med. 2019 Jul 25; 381(4): 317-327. doi: 10.1056/NEJMoa1903387.

44. O'Reilly E.M., Lee J.W., Zalupski M. et al. Randomized, Multicenter, Phase II Trial of Gemcitabine and Cisplatin With or Without Veliparib in Patients With Pancreas Adenocarcinoma and a Germline BRCA/ PALB2 Mutation. J Clin Oncol. 2020 Jan 24: JCO1902931. doi: 10.1200/JCO.19.02931.

45. Francois Y., Nemoz C.J., Baulieux J. et al. Influence of the interval between preoperative radiation therapy and surgery on downstaging and on the rate of sphincter-sparing surgery for rectal cancer: the Lyon R90-01 randomized trial. J Clin Oncol. 1999 Aug; 17(8): 2396. doi: 10.1200/JCO.1999.17.8.2396.

46. Probst C.P., Becerra A.Z., Aquina C.T. et al. Extended intervals after neoadjuvant therapy in locally advanced rectal cancer: the key to improved tumor response and potential organ preservation. J Am Coll Surg. 2015 August; 221(2): 430-440. doi: 10.1016/j.jamcollsurg.2015.04.010.

47. Lee S.W., Lee J.H., Lee I.K. et al. The impact of surgical timing on pathologic tumor response after short course and long course preoperative chemoradiation for locally advanced rectal adenocarcinoma. Cancer Res Treat. 2018; 50(3): 1039-1050. doi: 10.4143/crt.2017.252.

48. On J., Aly E.H. 'Watch and wait' in rectal cancer: summary of the current evidence. Int J Colorectal Dis. 2018 Sep; 33(9): 1159-1168. doi: 10.1007/s00384-018-3116-5.

49. Kwak Y.K., Kim K., Lee J.H. et al. Timely tumor response analysis after preoperative chemoradiotherapy and curative surgery in locally advanced rectal cancer: A multi-institutional study for optimal surgical timing in rectal cancer. Radiother Oncol. 2016 Jun; 119(3): 512-8. doi: 10.1016/j.radonc.2016.03.017.

50. Lefevre J.H., Mineur.L, Cachanado M. et al. Does a longer waiting period after neoadjuvant radiochemotherapy improve the oncological prognosis of rectal cancer? Three-year follow-up results of the GRECCAR- 6 randomized multicenter trial. J Clin Oncol. 37, 2019 (suppl 4; abstr 483). doi: 10.1097/SLA.0000000000003530.

51. Grothey A., Sobrero A.F., Shields A.F. et al. Duration of Adjuvant Chemotherapy for Stage III Colon Cancer. N Engl J Med. 2018; 378: 1177-1188. doi: 10.1056/NEJMoa1713709.

52. Andre T., Boni C., Navarro M. et al. Improved overall survival with oxaliplatin, fluorouracil, and leucovorin as adjuvant treatment in stage II or III colon cancer in the MOSAIC trial. J Clin Oncol. 2009; 27(19): 3109-3116. doi: 10.1200/JCO.2008.20.6771.

53. Iveson T., Sobrero A.F., Yoshino T. et al. Prospective pooled analysis of four randomized trials investigating duration of adjuvant (adj) oxaliplatin-based therapy (3 vs 6 months $\{\mathrm{m}\})$ for patients (pts) with high-risk stage II colorectal cancer (CC). J Clin Oncol. 37, 2019 (suppl; abstr 3501). doi: 10.1200/JCO.2019.37.15_suppl.3501.

54. Twelves C., Wong A., Nowacki M.P. et al. Capecitabine as adjuvant treatment for stage III colon cancer. N Engl J Med. 2005 Jun 30; 352(26): 2696-704. doi: 10.1056/NEJMoa043116.

55. De Gramont A., Bosset J-F., Milan C. et al. Randomized trial comparing monthly low-dose leucovorin and fluorouracil bolus with bimonthly high-dose leucovorin and fluorouracil bolus plus continuous infusion for advanced colorectal cancer: French intergroup study. J Clin Oncol. 1997; 15: 808-815. doi: 10.1200/ JCO.1997.15.2.808.

56. Fedyanin M.Y., Tryakin A.A., Tjulyandin S.A. Does asymptomatic primary tumor need to be removed in unresectable metastatic colon cancer? Pelvic Surgery and Oncology. 2015; 5(3): 9-14. (In Russ). doi: 10.17650/22203478-2015-5-3-9-14.

57. Alawadi Z., Phatak U.R., Hu C.Y. et al. Comparative effectiveness of primary tumor resection in patients with stage IV colon cancer. Cancer. 2017 Apr 1; 123(7): 1124-1133. doi: 10.1002/cncr.30230.

58. Fujitani K., Yang H.K., Mizusawa J. et al. Gastrectomy plus chemotherapy versus chemotherapy alone for advanced gastric cancer with a single non-curable factor (REGATTA): a phase 3, randomised controlled trial. Lancet Oncol. 2016 Mar; 17(3): 309-18. doi: 10.1016/S1470-2045(15)00553-7.

59. Tosello G., Torloni M.R., Mota B.S. et al. Breast surgery for metastatic breast cancer. Cochrane Database Syst Rev. 2018 Mar 15; 3: CD011276.

60. Méjean A., RavaudA., Thezenas S. et al. Sunitinib Alone or after Nephrectomy in Metastatic Renal-Cell Carcinoma. N Engl J Med. 2018 Aug 2; 379(5): 417-427. doi: 10.1056/NEJMoa1803675.

61. Kanemitsu Y., Shitara K., Mizusawa J. et al. A randomized phase III trial comparing primary tumor resection plus chemotherapy with chemotherapy alone in incurable stage IV colorectal cancer: JCOG1007 study (iPACS). J Clin Oncol. 2020; 38 (suppl 4; abstr 7). doi: 10.1093/jjco/hyz173.

62. Koppe M.J., Boerman O.C., Oyen W.J., Bleichrodt R.P. Peritoneal carcinomatosis of colorectal origin: incidence and current treatment strategies. Ann Surg. 2006; 243: 212-222. doi: 10.1097/01.sla.0000197702.46394.16.

63. Klaver Y.L., Lemmens V.E., Nienbuijs S.W., Luyer M.D., de Hingh I.H. Peritoneal carcinomatosis of colorectal origin: Incidence, prognosis and treatment options. World J Gastroenterol. 2012; 18: 5489-5494. doi: 10.3748/wjg.v18.i39.5489.

64. Elias D., Gilly F., Boutitie F., Quenet F., Bereder J.M., Mansvelt B., Lorimier G., Dubu P., Glehen O. Peritoneal colorectal carcinomatosis treated with surgery and perioperative intraperitoneal chemotherapy: retrospective analysis of 523 patients from a multicentric French study. J Clin Oncol. 2010; 28: 63-68. doi: 10.1200/JCO.2009.23.9285. 
65. Verwaal V.J., Bruin S., Boot H., van Slooten G., van Tinteren H. 8-year follow-up of randomized trial: cytoreduction and hyperthermic intraperitoneal chemotherapy versus systemic chemotherapy in patients with peritoneal carcinomatosis of colorectal cancer. Ann Surg Oncol. 2008; 15: 2426-2432. doi: 10.1245/s10434-008-9966-2.

66. Quene F., Elias D., Roca L. et al. A UNICANCER phase III trial of hyperthermic intra-peritoneal chemotherapy (HIPEC) for colorectal peritoneal carcinomatosis (PC): PRODIGE 7. Journal of Clinical Oncology. 2018; 36(no. 18 suppl). doi: 10.1200/JCO.2018.36.18_suppl.LBA3503.

67. Klaver C.E.L., Wisselink D.D., Punt C.J.A. et al. Adjuvant HIPEC in patients with colon cancer at high risk of peritoneal metastases: Primary outcome of the COLOPEC multicenter randomized trial. J Clin Oncol. 2019; 37 (suppl 4; abstr 482).

68. Yuan Z-X., Wang X-Y., Qin Q-Y. et al. The Prognostic Role of BRAF Mutation in Metastatic Colorectal Cancer Receiving Anti-EGFR Monoclonal Antibodies: A Meta-Analysis. PLoS ONE. 2013; 8(6): e65995. doi: 10.1371/journal. pone. 0065995 .

69. Mao C., Liao R.Y., Qiu L.X., Wang X.W., Ding H., Chen Q. BRAF V600E mutation and resistance to anti-EGFR monoclonal antibodies in patients with metastatic colorectal cancer: a meta-analysis. Molecular Biology Reports. 2011; 38: 2219-23. doi: 10.1007/s11033-013-2974-8.

70. Qi X.U., An Tao X.U., Ming Ming Z.H.U. et al. Predictive and prognostic roles of BRAF mutation in patients with metastatic colorectal cancer treated with anti-epidermal growth factor receptor monoclonal antibodies: A metaanalysis. Journal of Digestive Diseases. 2013; 14; 409-416. doi: 10.1111/1751-2980.12063.

71. Kopetz S., Desai J., Chan E. et al. PLX4032 in metastatic colorectal cancer patients with mutant BRAF tumors. J Clin Oncol. 2010; 28(15): suppl 3534. doi: 10.1200/jco.2010.28.15_suppl.3534.

72. Fedyanin M.Y., Tryakin A.A., Tjulandin S.A. Promises for treating coloc cancer patients with BRAF gene mutation. Pelvic Surgery and Oncology. 2014; (3): 9-16. (In Russ). doi: 10.17650/2220-3478-2014-0-3-9-16.

73. Kopetz S., Grothey A., Yaeger R. et al. Encorafenib, Binimetinib, and Cetuximab in BRAF V600E-Mutated Colorectal Cancer. N Engl J Med. 2019 Oct 24; 381(17): 1632-1643. doi: 10.1056/NEJMoa1908075. 